\title{
Les Audits Des Programmes ILDH : Les Balises D’une Évaluation Des Politiques Publiques Au Maroc
}

\author{
Mr. Mohammed Belga
}

Doctorant en droit public et sciences politiques Ả La Faculté Des Sciences Juridiques, Economiques Et Sociales De Rabat Agdal/Administrateur à La

Trésorerie Générale Du Royaume Maroc

\author{
Mr. Anass Kihli
}

Doctorant À La Faculté Des Sciences Juridiques, Economiques Et Sociales d'Oujda/ Inspecteur à La Direction Générale Des Impôts Maroc

\begin{abstract}
Policy assessment in Morocco is a recent method to measure the performance of public budget. It is considred as a new form of control of the effictiveness and efficiency of goverment expenditure. However, polemics have intensified about the content and the method of this new practice of control. The conception of an institutional framework of evaluation of public policy evaluation, has stood up to the multiplicity of its stakeholders. The double identity of the evaluative approach, proclaimed by public administration and parliament, made the definition of this practice problematic. Recently, The initiation of the ILDH programmes performance audit recently, has given gave a new path to follow, so as to get to the reality of the goverment evaluative action in Morocco.
\end{abstract}

Keywords: Public Management, Financial public governance, performance budget

\section{Résumé}

L'évaluation des politiques publiques (EPP) au Maroc est une pratique récente, qui vise la mesure de la performance du budget public. Il s'agit d'une nouvelle méthode de contrôle de l'efficacité et de l'efficience des dépenses de l'Etat. Or, l'institutionnalisation de la démarche évaluative s'est confrontée à plusieurs remous, qui ont entravé la définition claire de ses méthodes et des instances auxquelles sera confiée cette mission. La multitude des parties prenantes et la double identité, gestionnaire et politique, de l'EPP ont fait que son institutionnalisation soit problématique. Pourtant, l'amorcement, 
récemment, de l'audit de performance des programmes ILDH, semble avoir mis sur les rails la possibilité d'une réflexion savante sur les contours de la pratique évaluative au Maroc.

Mots-clés : Management public, Gouvernance financière publique, Le budget performance

\section{Introduction}

L'évaluation des politiques publiques est devenue, ces dernières années, au Maroc comme partout ailleurs, au cœur des débats sur la gestion de l'Etat et des collectivités territoriales. Trouvant ses origines dans le courant dit du Nouveau Management Public (NMP), elle constitue une consécration des principes de démocratie et de bonne gouvernance; dans la mesure où elle jalonne une nouvelle forme de contrôle sur les décideurs et gestionnaires publics, dans un contexte de crise de l'Etat providence, renforçant, par là, les principes de sincérité et de reddition des comptes.

Le déficit cyclique de la gestion publique, doublé d'une ascension fulgurante des exigences socioéconomiques, ont fait que les Etats se trouvent enclins de revoir leurs systèmes de gouvernance, balisant, ainsi, la voie à l'avènement du NMP comme paradigme de réforme, autour duquel s'articulent de nouveaux modèles de budgétisation et des méthodes de management traditionnellement étranges au secteur public. En effet, l'ancrage d'une culture des résultats dans la gestion publique, qui marque le passage d'une administration des procédures à une autre des performances, a engendré une reconfiguration du contrôle de l'action publique, qui est passé d'une logique de vérification de la régularité à une logique de mesure de la performance et des réalisations quantifiables. La réinvention du contrôle s'est traduite, également, par l'inclusion d'un souci d'amélioration de la gestion et de maîtrise des opérations dans la démarche du contrôleur ; d'où la notion du contrôle partenarial ou, pour reprendre l'expression de $\mathrm{COHEN}^{6}$, le contrôle sous forme de «boucle"; qui véhicule une philosophie de correction par erreur, par opposition au contrôle « linéaire » traditionnel axé sur la règle et sa sanction.

C'est dans ce contexte qu'est née l'évaluation des politiques publiques qui nous retient dans cet article. Elle se présente comme une nouvelle méthode de contrôle de l'action publique, qui vise le renforcement de la responsabilisation des décideurs et gestionnaires; désormais tenus de rendre compte de la pertinence de leurs choix, de la rationalité de leurs projets et de la performance de leur action.

${ }^{6}$ COHEN (A), Contrôle Interne et Audit Publics, LGDJ, Montchrestien, 2005, p. 22. 
Or, le consensus sur l'utilité de l'évaluation des politiques publiques n'a pas débouché sur une uniformisation de ses méthodes et outils, ni, encore moins, sur l'adoption d'une définition internationalement reconnue de ce concept $^{7}$, ce qui revient à dire que nous somme en présence d'une notion polysémique ; du fait d'une appréhension positiviste qui n'en embrasse pas toutes les facettes. En effet, le flou des définitions est le produit d'un lien imprécis entre l'évaluation et son objet, qui est injustement réduit dans la mesure de la performance ; qui, en réalité, n'en constitue qu'une dimension parmi d'autres ${ }^{8}$.

Par contre, une approche constructiviste de l'évaluation des politiques publiques suppose une vision d'ensemble, qui prend en compte la pertinence des décisions politiques, la rationalité de la stratégie et la performance de l'action administrative. Le présent article se veut, donc, de mettre en exergue cette confusion majeure entre évaluation, qui implique une prise de hauteur par rapport à l'ensemble de la politique analysée, et l'audit de performance qui se limite à l'appréciation des réalisations économiquement quantifiables et contribue, par là, à la maîtrise de la dépense publique, par la recherche d'un meilleur rapport coût-efficacité (I). Ensuite, l'accent sera mis, à la lumière d'exemples pratiques, sur l'ancrage de l'audit de performance dans la démarche des ISC au Maroc, comme avatar de l'évaluation des politiques et programmes publics(II).

\section{La mesure de performance : une notion au cour de l'évaluation des politiques publiques}

L'appréhension du concept de l'évaluation à travers la notion de performance est, à notre sens, la démarche la plus efficace pour sortir de l'engrenage des définitions dont s'est engouffré la plupart des auteurs en la matière. En effet, la confusion qui enrobe le concept d'évaluation est que celleci s'apparente, de part sa technicité, à l'audit ${ }^{9}$. Or, cette équivoque se dissipe au moment où l'on se rend compte que la démarche évaluative est un phénomène éminemment politique; dans la mesure où elle implique un jugement de valeur sur l'action d'un département ou d'une collectivité territoriale, chose que ne permet pas la seule mesure des résultats quantifiables. Dans ce sens, l'évaluation des politiques publiques suppose une

\footnotetext{
${ }^{7}$ BOULANGER $(\mathrm{H})$, « Les institutions supérieures de contrôle à l'heure de la maîtrise des dépenses publiques », GEOECONOMIE, 2013/4 n 67 p. 216.

${ }^{8}$ Dans ce sens : BESSIRE (D), «Définir la performance », Comptabilité-Contrôle-Audit, 1999/2, Tome 5, pp. 127-150.

9 TROSA (S), «L'évaluation des politiques publiques », Les notes du benchmarcking international, Institut de l'Entreprise, 2003, p. 14.
} 
« vision macro ${ }^{10}$, où la mesure de performance ne constitue qu'une dimension de la réalité, à coté de la pertinence des choix opérés et de la rationalité des stratégies (1). Cependant, si en théorie la délimitation des contours de l'évaluation des politiques publiques semble être comme une évidence, la pratique en matière de son institutionnalisation et de la démarche de sa mise en œuvre a fait preuve de plusieurs imperfections, notamment l'implication d'une multitude d'acteurs, malgré le rôle croissant des ISC dernièrement (2).

\section{Cadrage conceptuel : les trois dimensions de l'évaluation des politiques publiques}

$\mathrm{Au}$ fond, le besoin de rationalisation de l'action publique fut à la base de l'instauration de la méthode d'évaluation, dans le cadre du NMP ${ }^{11}$, comme moyen de mesure du rapport entre les ressources budgétaires utilisées et les réalisations sur le terrain, ce qui la rend semblable à une démarche d'audit. En ce sens, la démarche évaluative vise la légitimation des choix opérés ; à travers la justification de l'usage de l'argent publique. Néanmoins, l'identité politique de l'évaluation est perceptible à travers le jugement de valeur qu'elle véhicule $^{12}$, qui transcende la mesure de performance à l'analyse des forces profondes dernière la politique en question, c'est-à-dire la pertinence du choix, exprimée par l'adéquation entre les objectifs annoncés d'une politique et les problèmes qu'elle est censée résoudre.

Ceci dit, et si l'on se livre à un exercice de capitalisation, l'évaluation d'une politique publique ${ }^{13}$, qui se présente comme un «acte de jugement qui vise à lui conférer une valeur $\gg{ }^{14}$, ne saurait se faire en dehors du triptyque de la pertinence, la cohérence et la performance. Ces trois critères d'évaluation correspondent chacun à une dimension de la politique publique ; de manière à ce que sera jugée la pertinence des choix pour lesquels ont optés les décideurs face à un besoin (A), la cohérence des projets matérialisant les choix opérés (B) et, finalement, la performance de l'administration qui se présente comme l'objet chargé d'exécuter les projets, tels que dessinés par les décideurs

${ }^{10}$ BOULANGER $(\mathrm{H})$, « Les institutions supérieures de contrôle à l'heure de la maitrise des dépenses publiques $»$, op. cit. p. 216.

11 LACOUETTE-FOUERE (C) \& LASCOUMES (P), «Les scènes multiples de l'évaluation »-les problèmes récurrents de son institutionnalisation-, LIEPP, Science Po Paris, Rapport mais 2013, p. 15.

12 TROSA (S), «L'évaluation des politiques publiques », Les notes du benchmarcking international, op. cit. p. 14.

${ }^{13}$ Une politique publique est définie comme «un programme d'action propre à une ou plusieurs autorités publiques ou gouvernementales », THOENIG (J-C), L'Analyse des Politiques Publiques, Cité par GRAWITZ (M) \& LECA (J), Traité de Science Politique, PUF, Tome IV, Paris, 1985, p. 6.

${ }^{14}$ BESSIRE (D), « Définir la performance », Comptabilité-Contrôle-Audit, op. cit. p. 136. 
politiques $(\mathbf{C})$. L'association des trois critères aux trois dimensions de la réalité d'une politique publique, permet de l'appréhender selon la figure «sujet-projet-objet» qui constitue, selon BESSIRE ${ }^{15}$, une méthode constructiviste de la démarche évaluative.

\section{La dimension subjective ou intentionnelle de la politique publique : la pertinence du choix}

La première phase du cycle de vie d'une politique, si l'on se permet l'expression, est celle de l'intention ou le désir d'un sujet, en l'occurrence le décideur, souvent de surmonter un problème économique ou social, par le biais d'une action. La subjectivité est, ici, entendue dans le sens des raisons profondes derrière le choix opéré, qui expliquent le pourquoi de la politique adoptée, exprimé en terme des préférences et des priorités, répertoriées en fonction d'un besoin, dont l'ampleur est définie selon la discrétion du décideur politique. A ce niveau, l'évaluation porte sur la mesure de la pertinence de la décision politique, c'est-à-dire à quel point le sujet a fait «le bon choix », en usant de sa capacité de ciblage pour atteindre le maximum de précision et d'exactitude.

Dans ce sens, le NMP fait la distinction entre la conception de la politique, qui relève de la responsabilité des décideurs et dont la pertinence est mesurée à l'aune des effets qu'elle produit, c'est-à-dire sa capacité à résoudre les problèmes que rencontrent les citoyens, et entre la mise en œuvre de cette même politique qui revient aux gestionnaires publics, ayant l'obligation de faire preuve d'une action administrative performante ${ }^{16}$. Entre ces deux dimensions de la politique publique, la cohérence de la stratégie de mise en œuvre reste un critère déterminant de la démarche évaluative.

\section{La dimension projective ou rationnelle de la politique publique : la cohérence de la stratégie}

La seconde étape d'une politique publique qui constitue la suite logique de la conception est celle de la planification. A ce stade, il est, évidemment, fait référence à la stratégie adoptée par le décideur, afin de matérialiser l'idée de sa politique dans un ou plusieurs projets. La dimension projective, ainsi exprimée dans des plans d'actions ou des projets, fait appel à la notion de rationalité ; c'est à dire à une sorte d'ingénierie d'organisation à même de créer un enchainement harmonieux entre les décisions politiques et leur projection dans la réalité.

\footnotetext{
${ }^{15}$ Idem. pp. 127-150.

${ }^{16}$ Dans ce sens : GALDEMAR (V), GILLES (L) et SIMON (M-O), Performance, Efficacité, Efficience: Les Critères de l'Evaluation des Politiques Publiques Sociales sont-ils Pertinents?, CAHIER DE RECHERCHE No 299, CREDOC, Décembre 2012, p. 10. (Disponible sur : www.credoc.fr).
} 
A ce niveau, le critère d'évaluation retenu est celui de la cohérence des stratégies d'action et si elles vont toutes dans le même sens ; qui est celui de l'intention du sujet qui a initié la politique publique en question. La cohérence implique les concepts d'harmonie et de cohésion de la dimension projective, appréhendée à un double niveau interne et externe. La cohérence interne dénote l'existence d'une interaction systémique entre les projets au sein d'une même politique. La cohérence externe se traduit par l'inscription de cette politique dans le contexte d'autres actions et programmes publics et, par là, dans la stratégie globale du pays. A ce titre, CAPET affirme que la cohérence veut dire «que les décisions sont logiques entre elles et par rapport à une échelle de préférences $\gg{ }^{17}$.

Quoi qu'il en soit, la projection de la décision politique dans la réalité, telle que conçue et ordonnancée, suppose, alors, une conciliation entre pertinence du choix et cohérence de la démarche stratégique incombant aux décideurs. Or, une saine mise sur les rails des projets est hypothéquée par l'existence d'un appareil gestionnaire performant.

\section{La dimension objective de la politique publique : la performance de la gestion administrative}

Arrivant au stade de l'exécution de la décision politique, le point est mis sur l'administration publique, qui constitue 1'objet dont dispose les décideurs pour mettre en cuvre leurs projets. Il s'agit de la dimension gestionnaire et objective de la politique publique ; c'est-à-dire la poursuite des finalités fixées au départ et dessinées dans des plans. C'est le passage à l'action et à la mise en œuvre, qui ne doit pas sortir du cadre des lignes directrices tracées par les concepteurs de la politique. C'est, en d'autres termes, les réalisations économiquement quantifiables de l'action administrative, qui met en relation les ressources déployées et les objectifs atteints. L'originalité de cette étape réside dans le fait qu'elle forme le volet pratique ou opérationnel de la projection, ce qui en fait, d'ailleurs, le noyau dur de la politique publique dans son ensemble. C'est, en quelque sorte, le point de rencontre entre le décideur et les destinataires de la décision; qui s'attendent à des prestations tangibles. Ce constat fait que la politique publique est, en gros, jugée à travers sa dimension objective ; ce qui s'explique, à notre sens, par le fait que c'est à ce niveau que sont fournis les éléments d'appréciation les plus évidents; à savoir les résultats.

Ainsi présentée, la dimension objective de la politique publique est évaluée à la lumière de la notion de performance, qui constitue l'unité de mesure des objectifs et résultats. Ce critère se trouve, de par son caractère

${ }^{17}$ CAPET (M), CAUSSE (G) et MEUNIER (J), Diagnostic, organisation et planification d'entreprise, ECONOMICA, collection "Gestion", tome 1 : Diagnostic et politique générale, 2ème édition, Paris, 1986, p. 333. 
quantifiable, au cœur de la démarche évaluative. Par ailleurs, le poids du concept de performance est tel que cette dernière acquière une place de choix, au sein du nouveau paradigme de gestion dit NMP. En effet, la réforme de l'Etat et de la gestion publique dans sa globalité affiche la performance budgétaire comme étant son slogan phare, autour duquel se tisse toute l'action de l'administration.

La performance dans le secteur public est entendue dans le sens de l'optimisation des services rendus aux citoyens ${ }^{18}$, qui se manifeste par une augmentation d'accessibilité et de qualité, ainsi que par une réduction des coûts de production de ces services. Généralement, la mesure de la performance obéit à trois critères. En premier vient l'effectivité ; qui s'apprécie comme étant la distance entre prévision et mise en œuvre. Ensuite, l'efficacité s'établit comme le lien entre les moyens utilisés et les objectifs réalisés. Enfin, l'efficience s'annonce comme l'adéquation entre les ressources mobilisées et les résultats obtenus. Eu égard aux éléments quantifiables que fournie la mesure de performance, celle-ci se trouve au cœur de l'évaluation des politiques publiques, quitte à dire qu'elle en constitue une métamorphose ou une incarnation. Malgré l'adoption de la mesure de performance comme objet de l'évaluation des politiques et programmes publics, la question de son institutionnalisation fait au Maroc, comme d'ailleurs en France, l'objet de polémiques récurrentes.

\section{L'institutionnalisation de l'évaluation des politiques publiques : le rôle central des ISC}

$\mathrm{Au}$ fond, l'évaluation des politiques publiques a été conçue aux Etats Unis et aux pays anglo-saxons dans l'optique d'un contrepouvoir de l'exécutif, par le biais duquel le pouvoir législatif contrecarre l'omnipotence des gouvernements; en les tenant à légitimer l'usage des deniers publics. Ce constat fait, qu'en principe, c'est les assemblées parlementaires qui sont compétentes pour effectuer des évaluations de l'action publique, d'où la dimension politique de cette pratique. Or, l'existence d'un volet gestionnaire avéré de l'évaluation a fait que l'institution parlementaire se trouve dans l'impossibilité de se charger seule de cette besogne ; ce qui a acheminé vers la création d'organes spécialisés ${ }^{19}$ dans l'assistance du législateur dans cette mission.

\footnotetext{
${ }^{18}$ Dans ce sens : GALDEMAR (V), GILLES (L) et SIMON (M-O), Performance, Efficacité, Efficience: Les Critères de l'Evaluation des Politiques Publiques Sociales sont-ils Pertinents ?, op. cit. p. 9.

${ }^{19}$ Exemples du National Audit Office (NAO) crée en Grande Bretagne en 1960 et du vieux General Accounting Office (GAO) aux USA réformé en 1974, pour assurer des missions de conseil aux gouvernements et aux institutions parlementaires dans l'évaluation des politiques publiques.
} 
Inspirée de l'expérience étasunienne du General Accounting Office (GAO), la Cour des comptes en France s'est fixé l'objectif, depuis 1986, de devenir l'institution par excellence qui assiste le parlement en matière d'audit et d'évaluation des politiques publiques ${ }^{20}$, chose qu'elle obtient officiellement avec la constitution de $2008^{21}$. Au Maroc, la nouvelle constitution de 2011 est allée dans le même sens en stipulant que "la Cour des comptes assiste le Parlement dans le domaine de contrôle des finances publiques. Elle répond aux questions en rapport avec les fonctions de législation, de contrôle et d'évaluation, exercées par le Parlement et relatives aux finances publiques $»^{22}$. Au risque de raviver la polémique sur l'institutionnalisation de l'évaluation, obligation nous est faite d'affirmer que, malgré ces dispositions de la constitution, cette mission, matérialisée par l'audit de performance, est loin d'être l'apanage de la Cour des comptes. En effet, étant le fruit d'une tendance ou d'un courant réformateur du contrôle des finances publiques, une multitude d'institutions au Maroc, notamment les ISC, se sont assignées la mission d'effectuer des audits de performance des politiques et programmes publics, en adoptant cette pratique comme nouvelle orientation, reproduisant, par là, l'expérience de "l'indéfinissable approche à la française ${ }^{23}$ qui comprend un grand nombre d'acteurs, avec ses tares et vertus.

Outre le fait que l'implication d'une multitude d'acteurs dans la mission d'audit des programmes publics s'explique par un effet de mode, elle constitue une réponse aux carences des procédés traditionnels du contrôle parlementaire. De plus, la démarche évaluative comprend une composante technocratique indéniable à coté de son caractère politique ${ }^{24}$. La pratique d'évaluation s'est ancrée, également, comme un axe de réforme des Inspections Générales des Ministères qui ont orienté leur démarche, traditionnellement basée sur l'inspection, vers une fonction évaluative d'audit.

\section{L'ancrage de l'audit de performance dans la pratique des ISC comme avatar de l'EPP}

La réforme de la gestion publique s'est accompagnée d'une réingénierie du contrôle de l'exécution des dépenses de l'Etat et des collectivités territoriales. En effet, l'alignement des ISC sur la logique du NMP a balisé la voie à l'avènement d'une nouvelle forme de contrôle, qui ne

20 LACOUETTE-FOUERE (C) \& LASCOUMES (P), «Les scènes multiples de l'évaluation »-les problèmes récurrents de son institutionnalisation-, op. cit. p. 55.

${ }^{21}$ Article 47 / 2 de la constitution française de 2008.

${ }^{22}$ L'article 148 de la nouvelle constitution de 2011.

23 LACOUETTE-FOUERE (C) \& LASCOUMES (P), «Les scènes multiples de l'évaluation »-les problèmes récurrents de son institutionnalisation-, op. cit. p. 104.

${ }^{24}$ Dans ce sens : ESTEBAN (M), «L’évaluation : un outil pour l'amélioration de la gestion publique », in. Eléments d'analyse sur le développement territorial, Aspect théoriques et pratiques, l'Harmattan, Paris, 2007, p. 188. 
se limite plus à la vérification de la régularité des opérations de dépenses, par une démarche d'inspection fondée sur la détection des manquements aux règles et leurs sanctions, mais la dépasse à une finalité de « maîtrise », qui correspond à un objectif d'amélioration de la gestion et d'optimisation des ressources (1). Le changement de la logique du contrôle de l'exécution des dépenses publique, s'est traduit par l'avènement de nouvelles méthodes et de nouvelles missions, notamment l'audit de performance des politiques et programmes publics, désormais assignées aux ISC (2).

\section{Le contrôle de la dépense publique : de la vérification à la maîtrise}

Etymologiquement, le mot contrôle est formé de «contre » et de « rôle », et prend le sens de vérification ${ }^{25}$. En matière de dépense publique, le « contre-rôle » signifie la tenue en double d'un registre qui contient une même information chez l'initiateur de la dépense et son contrôleur, afin que les collationnements mutuels entre ces deux acteurs permettent, par un retour d'information, d'examiner la régularité des actes de dépenses, et d'en relever « les écarts par rapport à la norme ou les atteintes aux principes de conformité aux lois $\gg{ }^{26}$. Ce système régulateur comprend trois protagonistes qui sont les ordonnateurs, initiateurs des actes de dépenses, les comptables publics qui exercent un contrôle systématique sur l'ensemble de ces actes et, finalement, les ISC qui interviennent postérieurement à l'exécution de la dépense pour détecter les irrégularités de la procédure.

Or, la réforme du contrôle de la dépense publique, qui constitue une facette de la refonte globale du système des finances publiques et de l'Etat dans son ensemble, a marqué le passage d'un " contrôle-vérification » à un «contrôle-maîtrise ». Cet « art difficile de maîtrise», pour reprendre l'expression de BOULLENGER ${ }^{27}$, comprend, à notre sens, deux volets. Le premier est formé de la maîtrise des opérations et de la sécurisation des processus, c'est-à-dire l'inclusion d'une dimension de contrôle interne dans la démarche du contrôleur, notamment le comptable public, et de celle de l'initiateur des actes de dépenses, en l'occurrence l'ordonnateur, dans un souci d'amélioration de la capacité de gestion et de maîtrise des risques liés à l'exécution des dépenses, dans un contexte d'allègement du contrôle comptable a priori et d'orientation du contrôle a posteriori vers une démarche d'audit. Le second volet du « contrôle-maîtrise », et c'est ce qui nous retient le plus dans cet article, est celui de la maîtrise de la dépense elle-même ; c'està-dire 1'optimisation des ressources déployées dans les projets matérialisant

\footnotetext{
${ }^{25}$ André BARILARI, Les contrôles financiers comptables, administratifs et juridictionnels des finances publiques, L.G.D.J, Paris, 2003, p. 17.

${ }^{26}$ Déclaration de Lima sur les lignes directrices du contrôle des finances publiques.

${ }^{27}$ BOULANGER $(\mathrm{H})$, « Les institutions supérieures de contrôle à l'heure de la maîtrise des dépenses publiques », op. cit. p. 209.
} 
les politiques publiques. En effet, la maîtrise de la dépense renvoie à la notion d'allocation performante des dépenses, mesurée à l'aune d'une évaluation objective des résultats obtenus, d'où la nouvelle mission assignée aux ISC d'effectuer des audits de performance des programmes publics.

\section{L'audit de performance des programmes publics : la rationalité économique appliquée à l'action publique}

Selon les Normes ISSAI ${ }^{28}$ publiées par l'INTOSAI ${ }^{29}$, notamment 3100

et son annexe, l'audit de performance remplit une fonction d'ordre démocratique, consistant à fournir au public et au parlement, en premier lieu, une information fiable et précise sur la gestion gouvernementale. Il tient, également, l'entité auditée au courant de sa capacité de gestion et contribue à son amélioration, par la localisation des zones d'ombre et la proposition des recommandations susceptibles de les éclaircir. Etant un élément d'analyse de l'activité administrative, l'audit de performance n'est pas censé contester le bien fondé des décisions politiques ; mais se focalise sur l'évaluation objective des opérations avec des méthodes qui lui sont propres (A). Au Maroc, les ISC ne sont pas sorties, à l'occasion des audits de performance des programmes de 1'Initiative Nationale pour le Développement Humain (INDH), du cadre tracé par les normes ISSAI dans ce domaine, en suivant une approche globale axée sur les résultats et la gestion des risques(B).

\section{L'audit de performance : une méthode objective et indépendante d'évaluation politiques et programmes publics.}

L'audit de performance est "l'examen indépendant et objectif des entreprises, systèmes, programmes ou organisations du secteur public, sous l'angle de l'un ou plusieurs des trois aspects que sont l'économie, l'efficience et l'efficacité, dans la perspective de réaliser des améliorations ${ }^{30}$. Son application aux programmes publics s'inscrit dans un contexte de passage des ISC d'un contrôle de régularité traditionnel à des activités évaluatives. Son originalité par rapport aux audits de conformité ou financiers est le fait qu'il ne s'intéresse pas aux comptes mais à l'activité de l'organisation. Il s'agit d'une évaluation objective des opérations effectuées dans le cadre d'une politique ou d'un programme public, qui cherche à définir si les actions entretenues sont appropriées ou, dans le cas contraire, identifier les raisons du disfonctionnement et proposer des améliorations.

\footnotetext{
${ }^{28}$ Les Normes internationales des institutions de contrôle des finances publiques.

${ }^{29}$ Organisation internationales des institutions supérieures de contrôle des finances publiques.

${ }^{30}$ INTOSAI, Lignes directrices sur l'audit de la performance : principes fondamentaux. Téléchargeable sur : http://www.intosai.org/fr/issai-executive-summaries/detail/article/issai3100-performance-audit-guidelines-key-principles.html
} 
Provenant du domaine de la gestion de l'entreprise privée, l'audit de performance est conçu dans l'optique de l'application d'une rationalité économique à l'action publique, par un exercice d'analyse et d'évaluation des trois éléments de la performance. Ainsi, une action économique suppose la minimisation des coûts; en ce sens que la dotation de l'organisation par les moyens nécessaires à son activité doit se faire aux moindre prix. Ensuite, l'efficacité de l'action publique implique l'atteinte des objectifs d'un programme ou d'une politique. Enfin, l'usage des moyens mis à la disposition de l'administration publique doit se faire d'une manière efficiente ; c'est-àdire dans le respect d'une exploitation meilleure des ressources disponibles et de façon à réaliser un équilibre minutieux entre la quantité des moyens utilisés et la qualité des résultats obtenus.

La quête de la réalité dans le cadre d'un audit de performance se fait à travers deux approches, qui peuvent être combinées. La première est axée sur les résultats et cherche à mesurer le degré d'atteinte des objectifs. La seconde est orientée vers l'analyse des risques qui ont entravé l'atteinte de ces objectifs et, là, il est fait référence au dispositif du contrôle interne en place et les moyens de son perfectionnement pour une meilleure efficacité.

\section{L'audit des programmes INDH au Maroc : la mesure de la performance et de la capacité de gestion administrative}

La mise en œuvre des programmes de l'INDH au Maroc a fait l'objet de plusieurs missions d'audit de performance, effectuées par des ISC, notamment l'Inspection générale de l'administration territoriale (IGAT) et l'Inspection générale des finances (IGF). Un rapport de synthèse des missions publié par un groupe de travail conjoint IGAT/IGF ${ }^{31}$, fait état de 70 rapports d'audit élaborés par ces deux institutions. Une lecture dans le contenu de ce rapport donne une idée claire sur la démarche d'évaluation suivie par les ISC au Maroc, et qui ne sort pas du cadre des lignes directrices tracées par l'INTOSAI.

En effet, les missions d'audit sont allées dans le sens d'une analyse de l'ensemble du système de gestion des programmes ILDH, en procédant, dans un premier lieu, à l'examen des états financiers relatifs aux projets, afin d'apprécier l'efficacité de la procédure d'allocation des crédits aux services ordonnateurs, afin de leurs permettre d'établir les programmes d'emploi des ressources allouées par projet. L'analyse des états financiers a porté, également, sur le processus d'engagement et démission des dépenses dans le cadre de chaque programme, dans le but de jauger l'écart entre la planification et la mise en œuvre des projets. Dans un second lieu, le travail d'évaluation s'est enchaîné sur la constatation, par le biais d'échantillons représentatifs, de

${ }^{31}$ Téléchargeable sur : http://omdh.hcp.ma/attachment/209761/ 
la matérialité des dépenses effectuées dans le cadre de chaque projet, et ce à travers l'observation de la conformité des états d'avancement des travaux, présentés par les services dépensiers, avec les taux des réalisations constatées sur le terrain.

Ensuite, l'appréciation des résultats quantifiables s'est doublée, dans un exercice de combinaison des approches d'audit de performance, tel que tracée par les normes ISSAI, par une analyse du dispositif de contrôle interne des entités chargées de la mise en œuvre des projets, dans l'optique d'identifier les risques qui ont entravé la réalisation des objectifs. A ce niveau, un intérêt particulier est accordé à l'environnement du contrôle interne, qui a essentiellement trait à l'éthique et la déontologie des parties prenantes, ainsi qu'à l'engagement des organes de gestion à un niveau raisonnable de compétence. Cet exercice a débouché, en gros, sur la constatation l'absence d'une cartographie des risques éminents susceptible de compromettre la réussite des opérations menées dans le cadre des projets INDH, tel que la défaillance des études techniques, le retard dans l'exécution des travaux et le manque de rigueur face aux malfaçons entretenues.

A travers cet exemple d'audit de performance des projets ILDH, il s'avère que cette novelle méthode d'évaluation objective de l'action de l'administration, dans le cadre de l'exécution des programmes publics tels que tracées par les décideurs politiques, remplit une double fonction. La première consiste à mesurer le degré d'atteinte des objectifs tracés dans des plans et la seconde œuvre, par un effort d'analyse des dispositifs de contrôle interne en place, à l'identification et à la neutralisation des risques liées à l'exercice de l'activité administrative dans le cadre de l'exécution des projets, contribuant, par là, à l'amélioration de la capacité aux seins des administrations publiques.

\section{Conclusion}

Arrivant au stade de la conclusion, il est à affirmer que l'évaluation des politiques publiques au Maroc est une fonction récente, qui a hérité beaucoup des caractéristiques de l'expérience française dont elle s'est largement imprégnée, notamment une place de choix accordée à la mesure de performance et une démarche à multiples acteurs. En effet, étant un phénomène éminemment politique, l'évaluation est conçue dans l'optique d'une double fonction de légitimation de l'usage des finances publiques et d'un contrepouvoir de l'exécutif. En principe, cette mission suppose une vision d'ensemble par rapport à la politique analysée, qui s'articule autour des notions de pertinence de cohérence et de performance. Ce triptyque met en relation les décideurs, qui se livrent à un travail de conception et les gestionnaires qui exécutent les politiques concrétisées dans des projets. Or, en pratique, l'évaluation des politiques et programmes publiques ne sort pas du cadre de la gestion budgétaire axée sur les résultats et de la mesure de 
performance qui en découle ; c'est-à-dire l'établissement d'un lien direct entre la dépense publique et les réalisations quantifiables qu'elle a générées.

Partant de ce constat, l'implication des ISC en matière d'évaluation des politiques et programmes publiques est venue comme expression d'une volonté de renforcer les principes de bonne gouvernance financière, en incitant des institutions autres que le parlement à participer à une fonction qui est politique au fond, ce qui consacre une incarnation de l'évaluation dans l'audit de performance considéré comme un paradigme de réforme des ISC. Cela dit, l'efficacité de cette démarche à multiples acteurs reste hypothéquée par le fait que si cette dynamique évaluative des politiques publiques est l'expression d'une vision stratégique de l'Etat; ou si elle ne dépasse pas le stade d'un effet de mode passager.

\section{References:}

\section{OUVRAGES}

1. RENARD (J) et NUSSBAUMER (S), Audit interne et contrôle de gestion -pour une meilleure collaboration-, EYROLLYS, 2011.

2. COHEN (A), Contrôle Interne et Audit Publics, LGDJ, Montchrestien, 2005.

3. GUILlaUmE (H), DUREAU (G) et SILVENT (F), Gestion publique : 1'Etat et la performance, Presses de Sciences Po et DALLOZ, Paris, 2002.

4. SPENLEHAUER (V), L'évaluation des politiques à usages sociaux Trois études de cas d'évaluation-, l'Harmattan, Paris.

5. M. Bouvier, M.-C. Esclassan, J.-P. Lassale, Finances publiques, 10e édition, LGDJ, Paris, 2010.

6. BERRADA (M), L'audit interne tout simplement, AFRIQUE CHALLENGE EDITIONS, Casablanca, 2012.

7. MDERS (H) et MASSELIN (J), Contrôle interne des risques, Editions d'Organisation, Paris, 2004.

8. DEFARGES Philippe Moreau, La gouvernance, PUF, col. Que sais-je, 2003.

9. André BARILARI, Les contrôles financiers comptables, administratifs et juridictionnels des finances publiques, L.G.D.J, Paris, 2003.

10. CORDEL (F), Gestion des risques et contrôle interne, Vuibert, Paris, 2013.

11. BARTOLI (A), Management dans les organisations publiques, DUNOD, 3ème édition, 2010.

12. MDERS (H) et MASSELIN (J), Contrôle interne des risques, Editions d'Organisation, Paris, 2004. 


\section{ARTICLES}

1. Jean-Claude Barbier, «Éléments pour une sociologie de l'évaluation des politiques publiques en France ", Revue française des affaires sociales 2010/1 (n $\left.{ }^{\circ} 1-2\right)$, p. 25-49.

2. FOUQUET (A), «Méthodes d'évaluation des politiques publiques : actes du séminaire », ouvrage collectif, édition DREES, France, 2011, pp. 9 à 16.

3. THEONING (JC) et DURAN (P), «L'Etat et la gestion publique territoriale $»$, Revue française de science politique, $\mathrm{N}^{\circ}$ 4, Vol. 46, 1996, pp. 580-623.

4. BESSIRE (D), «Définir la performance », Comptabilité-ContrôleAudit, 1999/2, Tome 5, pp. 127-150.

5. JESSOP (B), «Essor de la gouvernance et ses risques d'échecs : le cas du développement économique », in Revue internationale des sciences sociales, $n^{\circ} 155$, mars 1998 , pp. 33-34.

6. DUFOUR $(\mathrm{C})$, «La réforme britannique après 20 ans : éléments de bilan ", in Télescope, vol. $7, \mathrm{n}^{\circ} 3$ septembre 2000 , pp. 1 à 9. (Disponible sur: http://www.enap.ca/enap-fra/observatoire/obstelescope.html).

7. Entre autres : HEWITT DE ALCANTARA (C), « Du bon usage du concept de gouvernance », Revue internationale des sciences sociales, $\mathrm{n}^{\circ} 155$, mars 1998, pp. 109 - 118.

8. Voir dans ce sens: SMOUTS (M-C), «Du bon usage de la gouvernance en relations internationales », in Revue internationale des sciences sociales, $\mathrm{n}^{\circ} 155$, mars 1998. pp. 85 à 94.

9. SCHICK (A), « Vingt-cinq années de réforme budgétaire », Revue de l'OCDE sur la gestion budgétaire, vol. 4 n 1, 2004. pp. 93 - 120. (Disponible sur : http://econpapers.repec.org/article/oecgovkab/519sr2xf0030.htm).

10. L'OCDE, «Transparence budgétaire : les meilleures pratiques de l'OCDE », Revue de l'OCDE sur la gestion budgétaire, vol. $1 \mathrm{n}^{\circ} 3$, $2002 . \quad$ Disponible sur: (http://www.oecd.org/fr/gov/budgetisation/Transparence\%20budg\% C3\%A9taire\%20meilleures\%20pratiques\%20COMPLET.pdf)

11. CULITINAUX $(\mathrm{J})$, « Nouveau management public et sujet critique : enjeux idéologiques, collectifs et subjectifs ", Nouvelle revue de psychosociologie, 2012/1 n ${ }^{\circ}$ 13, p. 195-207.

12. NIOCHE (J-P), «Science administrative, management public et analyse des politiques publiques », Revue Française d'Administration Publique, $\mathrm{n}^{\circ}$ 24, octobre/décembre, 1982, pp. 635-649. 
13. Dans ce sens : LAURENCE (M), « Un précurseur du New Public Management : Henri Fayol (1841-1925) », Gestion et management public 2012/2 (Volume $1 / \mathrm{n}^{\circ} 2$ ), pp. 4-21.

14. DAUPHIN (S), 2011, "Théorie du management », Informations sociales, 2011/5, n' 167 , pp. 6-9.

15. CHELLE (E), «Un militantisme réformateur : les manuels du nouveau management public », 